\title{
Alterations in functional connectivity and interactions in resting-state networks in female patients with functional constipation
}

\section{Lei Zhang}

Xidian University

Yuyao Liu

Air Force Medical University Xijing Hospital: Xijing Hospital

Guanya Li

Xidian University

\section{Xiaofang Yi}

Air Force Medical University Xijing Hospital: Xijing Hospital

\section{Yang Hu}

Xidian University

\section{Zongxin Tan}

Xidian University

Zhenzhen Jia

Xidian University

Hao Li

Xidian University

Shuai Lv

Xidian University

Karen M. von Deneen

Xidian University

Junwang Zhang

Air Force Medical University Xijing Hospital: Xijing Hospital

\section{Shijun Duan}

Air Force Medical University Tangdu Hospital

Guangbin Cui ( 861537506@qq.com )

Air Force Medical University Tangdu Hospital

Yi Zhang ( $\nabla$ zyrisk@163.com)

Xidian University https://orcid.org/0000-0003-0859-9735

Yongzhan Nie ( $\sim 1735503189 @ q q . c o m$ )

Air Force Medical University Xijing Hospital: Xijing Hospital 


\section{Research Article}

Keywords: functional constipation, functional connectivity, independent component analysis, restingstate $\mathrm{fMRI}$, resting-state network

Posted Date: May 5th, 2021

DOl: https://doi.org/10.21203/rs.3.rs-451507/v1

License: (c) (i) This work is licensed under a Creative Commons Attribution 4.0 International License. Read Full License 


\section{Abstract}

Patients with functional constipation (FCon) have been reported with brain functional abnormalities. The associations between constipation symptoms and brain functional connectivity (FC) in these patients are not well understood. Here we used functional magnetic resonance imaging (MRI) to examine FC alterations between resting-state networks (RSNs) and their associations with constipation symptoms in patients with FCon. The investigation of these characteristics might help to reveal the underlying neural mechanisms. Functional MRI with independent component analysis was applied to investigate alterations in FC within and functional network connectivity (FNC) between RSNs including default mode(DMN), basal ganglia- (BGN), salience- (SN), left and right control executive-network (LCEN/ RCEN) in 39 female patients with FCon and 36 female healthy controls (HC). Patient Assessment of Constipation Quality of Life Scale (PAC-QOL) and Patient Assessment of Constipation Symptom Scale (PAC-SYM) were used to assess the constipation symptoms. Results showed that FCon patients had changed regional FC between different networks contributed to the abnormal FNCs among RSNs compared with HC. Patients with greater stool syndromes had increased FNC of BGN-SN and DMN-LCEN, and patients with greater worries/concerns and PAC-QOL total score had reduced FNC of SN-RCEN. The greater strength changes of $\mathrm{FC}$ in prefrontal and in parietal cortices were associated with higher negative emotion scores and greater rectal symptoms respectively. Our functional MRI suggested that FCon patients had altered FC within and interaction between RSNs and the brain FC changes were associated with constipation symptoms and altered emotions.

\section{Introduction}

Functional constipation (FCon) is a type of functional gastrointestinal disorder (FGID) (De Giorgio et al., 2015; Koppen et al., 2015) with a prevalence of 9.5\% (Koppen et al., 2018; Mugie et al., 2011). FCon occurs with a higher incidence rate in female patients than in males (2.1:1) (Mugie et al., 2011) that it is important to focus on the impact of FCon on their physical and mental health.

Patients with FGID have been reported to associate with brain functional and structural alterations (Blankstein et al., 2010; Kwan et al., 2005; Lee et al., 2017). One functional magnetic resonance imaging (MRI) study on patients with FCon exhibited increased brain activities involved with emotional modulation and decreased activities involved with somatic sensory processing (Zhu et al., 2016). Another structural MRI study reported that patients with FCon had diminished regional cortical thickness (Hu et al., 2020). One recent publication revealed sex difference in resting state brain activity (Jin et al., 2019). Subsequent seed correlation analysis showed that female FCon had weaker functional connectivity (FC) of insula (INS)-orbitofrontal cortex (OFC) than males, which was negatively correlated with the anxiety in female FCon and with abdominal distension in male FCon group (Jin et al., 2019). These MRI studies revealed the impact of FCon on brain functions and structures, particularly the sex-related FC differences in patients with FCon. 
The measures of FC within and interaction between resting-state networks (RSNs) have been shown a better technique to represent intrinsic functional organization of the brain (Kelly et al., 2008; Seeley et al., 2007). It has been shown in patients with FGID (Gupta et al., 2014; Hong et al., 2016; Icenhour et al., 2017; Liu et al., 2016) and obesity (Ding et al., 2020) that reported abnormal interactions among RSNs with disordered symptoms and emotion. One study in female patients with irritable bowel syndrome (IBS) suggested that visceral sensitivity was related to alterations in FC within RSNs associated with sensory processing and interoception (Icenhour et al., 2017). Patients with IBS had greater brain activations in regions implicated in affective and attentional regulation, and altered brain response during cued pain conditions (Hong et al., 2016). Patients with IBS also showed increased FC within the salience network (SN) (Gupta et al., 2014) and greater activation to rectal distention is associated with enhanced coupling of SN with the default mode network (DMN) and control executive network (CEN) in key brain regions (Liu et al., 2016). These studies revealed alterations in FC strength within RSNs and their association with symptoms in patients with FGID. To our knowledge, no study has been performed to examine the FCon symptoms associated abnormalities in RSNs.

In the current study, resting-state functional MRI (fMRI) with independent component analysis (ICA) was employed to examine FC strength within and functional network connectivity (FNC) (Jafri et al., 2008) between RSNs in 39 female patients with FCon and 36 female HC. Five RSNs, including the basal ganglia network (BGN), SN, left and right control executive-network (LCEN/ RCEN), and DMN were first identified, and FNC was then used to examine the interactions between these RSNs. We hypothesized that patients with FCon had altered FC within and interactions between RSNs and these alternations were associated with constipation symptoms.

\section{Materials And Methods}

\section{Participants}

The experimental protocol was approved by the Institutional Review Board of Xijing Hospital and was registered in the Chinese Clinical Trial Registry Center as: ChiCTR-OOB-15006347 (http: //www. chictr.org.cn). The experiments were conducted in accordance with the Declaration of Helsinki. Female patients with FCon were recruited from a clinical site at Xijing Hospital affiliated with the Fourth Military Medical University in Xi'an, China, and female healthy subjects were recruited from the local community. An experienced gastroenterologist diagnosed FCon based on Rome IV criteria (Drossman, 2016). The current study included FCon patients with a variety of major bowel habits including functional defecatory disorders, slow transit constipation, and a combination of the two types. FCon patients with the following symptoms were excluded, including medical/ mental /neurological disorders requiring immediate treatment, constipation after childbirth, pelvic floor muscle relaxation/ congenital giant colon/redundant sigmoid colon, and current medications that could affect the central nervous system. All the subjects received a complete physical exam with history and signed an informed consent form. Thus, 39 female patients with FCon (right-handed, age $37.3 \pm 1.7$ years) and 36 female healthy controls (right-handed, age $41.8 \pm 2.5$ years, Table 1 ) were included in the current study. 
Table 1

Demographic and clinical information of patients with functional constipation and healthy control subjects.

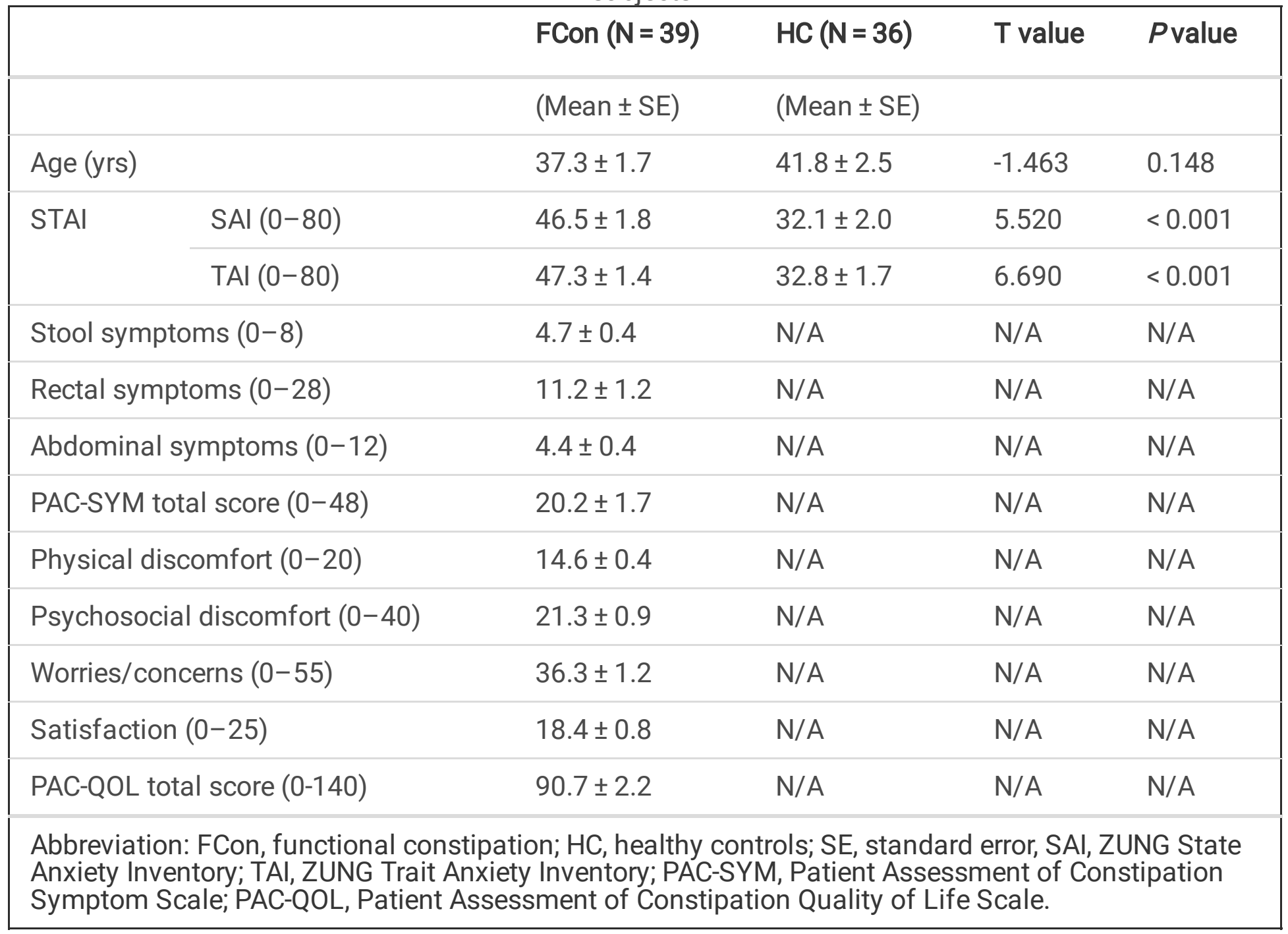

All participants were required to complete the state-trait anxiety inventory (STAI) to assess their severity of anxiety. They were also asked to complete the Patient Assessment of Constipation Symptom (PACSYM) (Frank, 1999) and Patient Assessment of Constipation Quality of Life Scale (PAC-QOL) (Marquis et al., 2005) to assess patients' constipation symptoms and quality of life.

\section{MRI acquisition}

The experiment was carried out using a 1.5T Signa HDXT (GE, Milwaukee, WI, USA) scanner. Parameters of the high-resolution three-dimensional T1-weighted imaging were as follows: TR $=9.1 \mathrm{~ms}, \mathrm{TE}=3.0 \mathrm{~ms}$, slice thickness $=1 \mathrm{~mm}$ and 248 slices, matrix size $=256 \times 256$, and field-of-view $=512 \times 512 \mathrm{~mm}^{2}$. Gradient echo $\mathrm{T} 2^{*}$-weighted echo planar imaging sequence was used to obtain resting-state functional images with the following parameters: TR $=2000 \mathrm{~ms}, \mathrm{TE}=40 \mathrm{~ms}$, field-of-view $=256 \times 256 \mathrm{~mm}^{2}$, matrix size $=$ $64 \times 64,29$ axial slices, flip angle $=90$ degree, and isotropic resolution of $4 \mathrm{~mm}^{3}$. The scan for resting-state fMRI lasted 400 seconds and participants were instructed to open their eyes during the scanning.

\section{Image processing}


The fMRI data were preprocessed using Statistical Parametric Mapping 12. The first five time points were removed to ensure magnetization equilibrium; and slice-timing and head movement correction were performed (Zhang et al., 2019). Then, the images were normalized to the Montreal-Neurological-Institute (MNI) template (voxel size of $3 \mathrm{~mm}^{3}$ ) (Friston et al., 1995) and smoothed with an isotropic Gaussian kernel (full-width-at-half-maximum $=6 \mathrm{~mm}^{3}$ ) (Yu et al., 2017). For the $\mathrm{FC}$ analysis, additional head motion parameters, global signals, white-matter signals, and cerebrospinal fluid were regressed out as nuisance covariates (Power et al., 2014). Scrubbing method (FD $>0.5 \mathrm{~mm}, \triangle B O L D$ of DVARS $>0.5 \%$ ) was used to remove the fMRI time points that were severely affected by motion, (Power et al., 2014) and less than $5 \%$ of time points were scrubbed for each subject. In the end, high-frequency noise and low frequency drift were eliminated using band-pass filter $(0.01-0.1 \mathrm{~Hz})$.

\section{Independent Component Analysis (ICA)}

The smooth fMRI data were analyzed by using the spatial group ICA (Calhoun, 2001) of the fMRI Toolbox (GIFT) software version 3.0b (Calhoun et al., 2009) to identify spatial independent and temporally coherent networks. First, resting-state fMRI data of 39 female patients with FCon and 36 female HC were concatenated by principal component analysis and 26 principal components were obtained by using a minimum description length approach. Then, the Infomax ICA algorithm (Bell and Sejnowski, 1995) was repeated 20 times in ICASSO to verify the robustness of the ICA decomposition. Finally, these 26 independent components (ICs) were individually backreconstructed for each participant. The resulting image maps and time courses were converted into z-scores. Calculating the spatial correlation between ICs and templates (Stanford's Functional Imaging in Neuropsychiatric Disorders Lab;

http://findlab.stanford.edu/ functional_ROls.html), we selected the components which showed highest correlation with the templates (Shirer et al., 2012).

\section{FNC analysis}

We used the FNC toolbox (TRENDS Center, version 2.3a) to calculate the temporal correlation between five RSNs (Jafri et al., 2008). The maximal lagged correlation approach that lag varied between -3 to 3 seconds at $3 / 25$ second intervals was conducted to estimate pairwise correlations between the five selected ICs (in total 10 combinations). The differences in FNC were tested via a two-sample $t$-test.

\section{Group difference within RSNs}

Among the 26 components, five components were selected as five RSNs, including DMN, BGN, SN, LCEN, and RCEN. For each RSN, a mask was defined by conducting a one-sample $t$-test of the Z-maps from the two groups. In order to calculate the differences between two groups, we performed a two-sample $t$-test $(P<0.05$, false wise error-FWE corrected) on the Z-maps of the five RSNs within the corresponding masks.

\section{FC analysis within RSNs with group differences in FNC}

Within the RSNs which showed group differences in FNC, the regions-of-interest (ROIs) which belong to the key hubs in RSNs were defined by conducting a one-sample $t$-test of the Z-maps from the two groups. The voxels centered at the Talairach coordinate with a peak $t$ score and its surrounding voxels within a 
radius of $6 \mathrm{~mm}$ were selected for further correlation analyses. Furthermore, the significance of group differences in pairwise correlations from all ROls was tested using two-sample t-test to assess abnormity within RSNs between patients with FCon and HC.

\section{Clinical measurement correlation}

Correlation analysis were used to calculate the association between imaging data and clinical measurements in patients with FCon. The FNC between the selected RSNs and their correlations with clinical measurements can be acquired. Bonferroni correction was used for multiple comparisons $(P<$ $0.002(0.05 / 27))$. On the other hand, the FC strengths between ROIs and their correlations with clinical measurements were also conducted. Bonferroni correction was used for multiple comparisons $(P<0.003$ $(0.05 / 18))$.

\section{Results}

\section{Demographic characteristics}

There was no significant difference in age between patients with FCon and HC groups $(P>0.05$, Table 1$)$. SAl and TAI were higher for patients with FCon than for $\mathrm{HC}(P<0.001$, Bonferroni corrected). PAC-SYM and PAC-QOL scores of the FCon group are also shown in Table 1.

\section{Identification of RSNs}

In the ICA analysis, 26 components were extracted from patients with FCon and HC groups. We calculated the spatial correlation between the ICs and the anatomical masks of each network to determine which ICs had the strongest spatial correlation with functional networks of interest (DMN, BGN, SN, LCEN, and RCEN). The ICs showed the highest correlation with these RSNs were IC17 (DMN, $r=$ 0.61991), IC5 (BGN, $r=0.13772), I C 15$ (SN, $r=0.53556)$, IC22 (LCEN, $r=0.63165)$, and IC19 (RCEN, $r=$ $0.55784)$.

\section{FNC analysis}

One-sample $t$-tests showed that ten connections with positive and significant correlation coefficients in both FCon and HC groups (Fig. 1A a, b). Same orientations of the arrow were observed in both groups (Fig. 1B a, b). Two-sample $t$-test revealed that two network connections of SN-BGN $(P=0.0032, \mathrm{t}=3.049)$ and DMN-LCEN $(P=0.0044, \mathrm{t}=2.938)$ had a significant stronger FNC in FCon than that in HC (Fig. 1A c). The SN-RCEN ( $P=0.0049, \mathrm{t}=-2.905)$ connection had a significant stronger $\mathrm{FNC}$ in $\mathrm{HC}$ than that in FCon (Fig. 1A c).

\section{FC analysis within RSNs}

Two-sample $t$-test of FNC showed significant differences in FNC in three connections of the SN-BGN $(P=$ $0.0032, \mathrm{t}=3.049)$, SN-RCEN $(P=0.0049, \mathrm{t}=-2.905)$, and DMN-LCEN $(P=0.0044, \mathrm{t}=2.938)$. Two-sample $t$ test on the Z-maps of the five RSNs within corresponding masks showed there was no significant 
difference between the two groups. Then, a one-sample $t$-test of the Z-maps from the two groups was performed in these five RSNs, and ROIs (center at the coordinates of the peak value with a $6 \mathrm{~mm}$ radius) which represent the key hubs of each RSN were defined correspondingly. For the DMN, ventromedial prefrontal cortex (VMPFC), precuneus (PCUN), left-(ANG_L), and right angular gyrus (ANG_R) were selected. For the SN, left-(INS_L) and right insula (INS_R), and ACC were selected. Left-(CAU_L) and right caudate (CAU_R), and left-(THA_L), and right thalamus (THA_R) were selected in the BGN. Left dorsolateral prefrontal cortex (DLPFC_L) and ANG_L were selected in the LCEN. For the RCEN, right DLPFC (DLPFC_R) and ANG_R were selected.

For the BGN-SN, SN-RCEN, and DMN-LCEN connections, the pair-wise FC strength consisting of a region $\times$ region matrix for each subject was calculated. In order to examine the differences between the two groups, an independent two sample $t$-test was conducted, and results are shown in Fig. 2 and Fig. 3. Within the BGN-SN connection, FCon compared with HC had higher pair-wise FC strengths of CAU_LINS_L, THA_R-INS_L, CAU_R-INS_L, and THA_L-ACC (Fig. 2A, P $<0.0024(0.05 / 21)$ ). Within the SN-RCEN connection, FCon showed lower pairwise FC strengths of INS_L-DLPFC_R and INS_R-DLPFC_R (Fig. 2B, P $<0.005(0.05 / 10))$. Within the DMN-LCEN connection, FCon showed higher pair-wise FC strengths of VMPFC-ANG_L, PCUN-ANG_L, and VMPFC-DLPFC_L (Fig. 3A, P<0.003 (0.05/15)).

\section{Correlation between imaging data and clinical measurement in FCon}

FNC of the SN-RCEN was negatively correlated with PAC-QOL total score ( $P=0.0001, \mathrm{r}=-0.5080$, Fig. 4$)$ and worries/concerns $(P=0.0043, \mathrm{r}=-0.4475)$ in FCon. There were positive correlations between $\mathrm{FNC}$ of the DMN-LCEN and stool symptoms of the PAC-SYM scale $(P=0.0003, r=0.5534$, Fig. 4$)$ in FCon

FC strength of VMPFC-DLPFC was not only positively correlated with worries/concerns of the PAC-QOL scale $(P=0.0002, \mathrm{r}=0.5564)$ but also with the PAC-QOL total score $(P=0.0013, \mathrm{r}=0.4964$, Fig. 3B $)$ in FCon. FC strength of PCUN-ANG_L was positively correlated with rectal symptoms of the PAC-SYM scale $(P=0.0031, r=0.4614)$ and PAC-SYM total score $(P=0.0033, r=0.4593$, Fig. $3 B)$ in FCon.

\section{Discussion}

In the current study, we employed resting-state FMRI with ICA to explore alterations in FC within and FNC between five RSNs in patients with FCon. We provided evidence that the FCon patients had altered FC within and interactions among RSNs. FC analysis within RSNs showed compared to heathy controls, the FCon patients had increased FNC of BGN-SN and DMN-LCEN and reduced FNC of SN-RCEN. In the subsequent $F C$ analysis to examine the interactions between RSNs, we found changes in regional $F C$ of the CAU_L-INS_L, THA_R-INS_L, CAU_R-INS_L, THA_L-ACC, INS_L-DLPFC_R, INS_R-DLPFC_R, VMPFCANG_L, PCUN-ANG_L, and VMPFC-DLPFC contributed to the abnormal FNC in FCon. The correlation analysis between RSN and clinical measures revealed FNC of SN-RCEN was negatively correlated with worries/concerns and the total score of the PAC-QOL scale, whereas FNC of the DMN-RCEN was positively correlated with stool symptoms of the PAC-SYM scale. FC strength of VMPFC-DLPFC_L was 
positively correlated with the worries/concerns and the PAC-QOL total score, whereas FC strength of PCUN-ANG_L was positively correlated with rectal symptoms of the PAC-SYM scale and PAC-SYM total score.

\section{Alterations in FNC between BGN and SN}

Our data showed significant group differences in three network connections in patients with FCon relative to HC, including increased FNC of BGN-SN, DMN-LCEN, and decreased FNC of SN-RCEN. The SN is a paralimbic network that deals with internal attention, cognition, emotion, and regulation functions (Menon and Uddin, 2010; Seeley et al., 2007). Visceral afferent signals reach the primary interoceptive cortex through the thalamus, regulating affective, motor and motivational brain response to visceral sensation (Menon and Uddin, 2010). Thalamus is the key node of the BGN, which plays an important role in perceptual processing (Sherman and Guillery, 2006). Our data showed strong FNC of BGN-SN in FCon, indicating the abnormality in visceral sensation and emotional regulation.

FC analysis between BGN and SN showed significant increases in FC strengths of CAU_L-INS_L, THA_RINS_L, CAU_R-INS_L, and THA_L-ACC in FCon compared with HC, contributing to the increased FNC of BGN-SN. The INS and ACC are the key nodes of SN. The INS has many functions in human beings, from sensory and emotional processing to advanced cognition (Uddin et al., 2017). The ACC plays a significant role in emotional response regulation, decision-making, and emotional responses to visceral stimulation (Cohen et al., 2005). The THA receives input from the basal ganglia nucleus and projects them to the prefrontal cortex to regulate cortical activity (Jakab et al., 2012). The increases in FC strength of THA_LACC in patients with FCon may reflect the increase of attention and regulatory resources of visceral afferent signals and emotional response (Icenhour et al., 2017). The homeostatic afferent network composed of THA, INS, and ACC can regulate visceral sensation and response, (Mayer et al., 2006) and the increases in FC strength of THA_R-INS_L in FCon might be associated with abnormal regulation of visceral sensation. The CAU is related to pain processing, (Borsook et al., 2010) and abnormal structure and function of CAU have been reported in IBS studies (Bhatt et al., 2019; Weng et al., 2017). The increases in FC strengths of CAU_L-INS_L and CAU_R-INS_L revealed abnormal pain processing.

\section{Alterations in FNC between SN and RCEN}

The abnormal interactions among SN, DMN, and CEN play an important role in understanding psychiatric and neurological disorders, including anxiety and depression (Menon, 2011). The decreased FNC between the SN and RCEN in patients with FCon may compromise the dynamic interaction of these networks. Our data showed FNC of SN-RCEN was negatively correlated with the worries/concerns and the PAC-QOL total score, reflecting the aberrant interaction between SN and RCEN might affect the negative emotions including anxiety and depression.

The DLPFC and ventrolateral prefrontal cortex, INS, and amygdala have impact on regulating the pain response via emotions such as anxiety and stress in patients with chronic pain (Boeckxstaens et al., 2016). One study reported that cognitive impairment in patients with IBS was related to the decreased 
activity in the DLPFC (Aizawa et al., 2012). Our data showed the lower pair-wise FC strengths of INS_LDLPFC_R and INS_R-DLPFC_R in FCon may contribute to abnormal emotion.

\section{Alterations in FNC between DMN and LCEN}

The DMN is involved in self-reference processing, including monitoring emotional state and physical condition, (Davey et al., 2016) and altered DMN connectivity has also been revealed in IBS studies (Qi et al., 2016). One study reported that FC of the INS and cingulate seeds both increased significantly in patients with IBS in the VMPFC, dorsomedial prefrontal cortex, and posterior cingulate cortex. These changes revealed excessive coupling of the SN with the CEN and DMN (Liu et al., 2016). Our data showed FCon patients had stronger FNC of DMN-LCEN. The stronger FNC is positively correlated with stool symptoms of the PAC-SYM scale. Our results suggested the abnormal interaction between DMN and LCEN might be associated with constipation symptoms.

The PCUN is the key hub of the DMN, which is related to the processing of self-referral information (Tomasi and Volkow, 2011). As a part of the DMN, the VMPFC may be related to the disturbed emotion regulation in IBS (Icenhour et al., 2019). The DLPFC has been implicated in attention control and chronic pain regulation (Lorenz et al., 2003). Current results showed FC strength of the VMPFC-DLPFC was positively related to the worries/concerns and the PAC-QOL total score in patients with FCon. The higher FC of the VMPFC-DLPFC was related to higher negative emotion scores, which will lead to abnormal emotion regulation. Our results suggested the FC strength of PCUN-ANG_L was positively correlated with rectal symptoms and PAC-SYM total score in patients with FCon. Previous study revealed the connectivity of the PCUN and ANG was strongly related to the efficiency of conducting an executive control attention task (Visintin et al., 2015). The aberrant FC of PCUN and ANG_L might be associated with more attention to the bowel movement of patients with FCon and as a consequence of negative emotions, which would worsen constipation symptoms.

\section{Conclusion}

The current study investigated alterations in FC within and FNC between RSNs in patients with FCon compared with HC. Our fMRI results revealed the FCon patients had increased interactions of the BGN-SN and DMN-LCEN and reduced interactions between SN and RCEN compared with HC. FNC analysis suggested that aberrant RSN interactions in the patients might be associated with the abnormality in emotion regulation and constipation symptoms. Changes in regional FC between different networks contributed to the abnormal FNCs among RSNs. In FCon patients, increased FNC of the DMN-LCEN was associated with stool syndromes and reduced FNC of the SN-RCEN was associated with worries/concerns and the PAC-QOL total score. FC strength changes in prefrontal and in parietal cortices were associated with worries/concerns and with rectal symptoms respectively. Our findings provided evidence that the FCon patients had altered FC within and interaction between RSNs in brain. The brain FC changes were associated constipation symptoms and altered emotions presented in the patients. 


\section{Declarations}

\section{Ethical approval}

All procedures performed in studies involving human participants were in accordance with the ethical standards of the institutional and/or national research committee and with the 1964 Helsinki Declaration and its later amendments or comparable ethical standards.

\section{Consent to participate}

Informed consent was obtained from all participants included in the study.

\section{Consent to publish}

All the authors agreed with the publication of this article.

\section{Authors contributions}

$Y N, Y Z$, and JZ were responsible for study concept/design. $Y N$ and $X Y$ were responsible for diagnosis. GC and SD were responsible for image data collection. $L Z, Y L, G L, Y H, Z T, Z J, H L$ and $S L$ assisted with data analysis/interpretation of findings. LZ and YL drafted the manuscript. KMD and YZ provided critical revision of the manuscript for important intellectual content. All authors critically reviewed the content and approved the final version for publication.

\section{Funding}

This work is supported by the National Natural Science Foundation of China under Grant Nos. 61431013, 81470816, 81730016; the Open Funding Project of National Key Laboratory of Human Factors Engineering under Grant No. 6142222190103; National Clinical Research Center for Digestive Diseases, Xi'an, China under Grant No. 2015BAI13B07.

\section{Competing interests}

The authors declare no conflict of interest.

\section{Availability of data and materials}

The datasets generated for this study are available on request to the corresponding author.

\section{References}

Aizawa, E., Sato, Y., Kochiyama, T., Saito, N., Izumiyama, M., Morishita, J., et al. (2012). Altered cognitive function of prefrontal cortex during error feedback in patients with irritable bowel syndrome, based on FMRI and dynamic causal modeling. Gastroenterology, 143(5), 1188-1198. 
Bell, A.J., Sejnowski, T.J. (1995). An information-maximization approach to blind separation and blind deconvolution. Neural Comput, 7(6), 1129-1159.

Bhatt, R.R., Gupta, A., Labus, J.S., Zeltzer, L.K., Tsao, J.C., Shulman, R.J., et al. (2019). Altered brain structure and functional connectivity and its relation to pain perception in girls with irritable bowel syndrome. Psychosom Med, 81(2), 146-154.

Blankstein, U., Chen, J., Diamant, N.E., Davis, K.D. (2010). Altered brain structure in irritable bowel syndrome: potential contributions of pre-existing and disease-driven factors. Gastroenterology, 138(5), 1783-1789.

Boeckxstaens, G., Camilleri, M., Sifrim, D., Houghton, L.A., Elsenbruch, S., Lindberg, G., et al. (2016). Fundamentals of neurogastroenterology: physiology/motility-sensation. Gastroenterology, 150(6), 12921304.

Borsook, D., Upadhyay, J., Chudler, E.H., Becerra, L. (2010). A key role of the basal ganglia in pain and analgesia - insights gained through human functional imaging. Mol Pain, 6(27).

Calhoun. (2001). A method for making group inferences from functional mri data using independent component analysis. Human Brain Mapping, 14 140-151.

Calhoun, V.D., Liu, J., Adalı, T. (2009). A review of group ICA for $\mathrm{fMRI}$ data and ICA for joint inference of imaging, genetic, and ERP data. Neuroimage, 45(1), S163-S172.

Cohen, M.X., Heller, A.S., Ranganath, C. (2005). Functional connectivity with anterior cingulate and orbitofrontal cortices during decision-making. Cognitive Brain Research, 23(1), 61-70.

Davey, C.G., Pujol, J., Harrison, B.J. (2016). Mapping the self in the brain's default mode network. Neuroimage, 132390-397.

De Giorgio, R., Ruggeri, E., Stanghellini, V., Eusebi, L.H., Bazzoli, F., Chiarioni, G. (2015). Chronic constipation in the elderly: a primer for the gastroenterologist. BMC Gastroenterol, 15(1), 130.

Ding, Y., Ji, G., Li, G., Zhang, W., Hu, Y., Liu, L., et al. (2020). Altered interactions among resting-state networks in individuals with obesity. Obesity, 28(3), 601-608.

Drossman, D.A. (2016). Functional gastrointestinal disorders: history, pathophysiology, clinical features, and Rome IV. Gastroenterology, 150(6), 1262-1279.

Friston, K.J., Ashburner, J., Frith, C.D., Poline, J.B., Heather, J.D., Frackowiak, R.S.J. (1995). Spatial registration and normalization of images. Human Brain Mapping, 3(3), 165-189.

Gupta, A., Kilpatrick, L., Labus, J., Tillisch, K., Braun, A., Hong, J., et al. (2014). Early adverse life events and resting state neural networks in patients with chronic abdominal pain: evidence for sex differences. 
Psychosom Med, 76(6), 404-412.

Hong, J.Y., Naliboff, B., Labus, J.S., Gupta, A., Kilpatrick, L.A., Ashe-McNalley, C., et al. (2016). Altered brain responses in subjects with irritable bowel syndrome during cued and uncued pain expectation. Neurogastroenterology \& Motility, 28(1), 127-138.

Hu, C., Liu, L., Liu, L., Zhang, J., Hu, Y., Zhang, W., et al. (2020). Cortical morphometry alterations in brain regions involved in emotional, motor-control and self-referential processing in patients with functional constipation. Brain Imaging Behav, 14(5), 1899-1907.

Icenhour, A., Tapper, S., Bednarska, O., Witt, S.T., Tisell, A., Lundberg, P., et al. (2019). Elucidating the putative link between prefrontal neurotransmission, functional connectivity, and affective symptoms in irritable bowel syndrome. SCI REP-UK, 9(1).

Icenhour, A., Witt, S.T., Elsenbruch, S., Lowén, M., Engström, M., Tillisch, K., et al. (2017). Brain functional connectivity is associated with visceral sensitivity in women with Irritable Bowel Syndrome. Neurolmage: Clinical, 15 449-457.

Jafri, M.J., Pearlson, G.D., Stevens, M., Calhoun, V.D. (2008). A method for functional network connectivity among spatially independent resting-state components in schizophrenia. Neuroimage, 39(4), 1666-1681.

Jakab, A., Blanc, R., Berényi, E.L. (2012). Mapping changes of in vivo connectivity patterns in the human mediodorsal thalamus: correlations with higher cognitive and executive functions. Brain Imaging Behav, 6(3), 472-483.

Jin, Q., Duan, S., Li, G., Sun, L., Hu, Y., Hu, C., et al. (2019). Sex-related differences in resting-state brain activity and connectivity in the orbital frontal cortex and insula in patients with functional constipation. Neurogastroenterology \& Motility, 31(5), e13566.

Kelly, A.M.C., Uddin, L.Q., Biswal, B.B., Castellanos, F.X., Milham, M.P. (2008). Competition between functional brain networks mediates behavioral variability. Neuroimage, 39(1), 527-537.

Koppen, I.J.N., Lammers, L.A., Benninga, M.A., Tabbers, M.M. (2015). Management of functional constipation in children: therapy in practice. Pediatr Drugs, 17(5), 349-360.

Koppen, I.J.N., Vriesman, M.H., Saps, M., Rajindrajith, S., Shi, X., van Etten-Jamaludin, F.S., et al. (2018). Prevalence of functional defecation disorders in children: a systematic review and meta-analysis. Journal of Pediatrics, 198, 121.

Kwan, C.L., Diamant, N.E., Pope, G., Mikula, K., Mikulis, D.J., Davis, K.D. (2005). Abnormal forebrain activity in functional bowel disorder patients with chronic pain. Neurology, 65(8), 1268-1277.

L. Frank, L.K.C.F. (1999). Psychometric validation of a constipation symptom assessment questionnaire. Scand J Gastroentero, 34(9), 870-877. 
Lee, I., Preissl, H., Enck, P. (2017). How to perform and interpret functional magnetic resonance imaging studies in functional gastrointestinal disorders. Journal of Neurogastroenterol and Motility, 23(2), 197207.

Liu, X., Silverman, A., Kern, M., Ward, B.D., Li, S.J., Shaker, R., et al. (2016). Excessive coupling of the salience network with intrinsic neurocognitive brain networks during rectal distension in adolescents with irritable bowel syndrome: a preliminary report. Neurogastroenterology \& Motility, 28(1), 43-53.

Lorenz, J., Minoshima, S., Casey, K.L. (2003). Keeping pain out of mind: the role of the dorsolateral prefrontal cortex in pain modulation. Brain, 126(Pt 5), 1079-1091.

Marquis, P., De La Loge, C., Dubois, D., McDermott, A., Chassany, O. (2005). Development and validation of the Patient Assessment of Constipation Quality of Life questionnaire. Scand J Gastroenterol, 40(5), 540551.

Mayer, E.A., Naliboff, B.D., Craig, A.D.B. (2006). Neuroimaging of the brain-gut axis: From basic understanding to treatment of functional G1 disorders. Gastroenterology, 131(6), 1925-1942.

Menon, V. (2011). Large-scale brain networks and psychopathology: a unifying triple network model. Trends Cogn SCl, 15(10), 483-506.

Menon, V., Uddin, L.Q. (2010). Saliency, switching, attention and control: a network model of insula function. Brain Structure and Function, 214(5-6), 655-667.

Mugie, S.M., Benninga, M.A., Di Lorenzo, C. (2011). Epidemiology of constipation in children and adults: A systematic review. Best Practice \& Research Clinical Gastroenterology, 25(1), 3-18.

Power, J.D., Mitra, A., Laumann, T.O., Snyder, A.Z., Schlaggar, B.L., Petersen, S.E. (2014). Methods to detect, characterize, and remove motion artifact in resting state fMRI. Neuroimage, 84 320-341.

Qi, R., Liu, C., Ke, J., Xu, Q., Ye, Y., Jia, L., et al. (2016). Abnormal amygdala resting-state functional connectivity in irritable bowel syndrome. AM J Neuroradiol, 37(6), 1139-1145.

Seeley, W.W., Menon, V., Schatzberg, A.F., Keller, J., Glover, G.H., Kenna, H., et al. (2007). Dissociable intrinsic connectivity networks for salience processing and executive control. The Journal of Neuroscience, 27(9), 2349-2356.

Sherman, S.M., Guillery, R.W. (2006). Exploring the thalamus and its role in cortical function. Cambridge, MIT Press.

Shirer, W.R., Ryali, S., Rykhlevskaia, E., Menon, V., Greicius, M.D. (2012). Decoding subject-driven cognitive states with whole-brain connectivity patterns. Cereb Cortex, 22(1), 158-165. 
Tomasi, D., Volkow, N.D. (2011). Association between functional connectivity hubs and brain networks. Cereb Cortex, 21(9), 2003-2013.

Uddin, L.Q., Nomi, J.S., Hébert-Seropian, B., Ghaziri, J., Boucher, O. (2017). Structure and function of the human insula. J Clin Neurophysiol, 34(4), 300-306.

Visintin, E., De Panfilis, C., Antonucci, C., Capecci, C., Marchesi, C., Sambataro, F. (2015). Parsing the intrinsic networks underlying attention: a resting state study. Behav Brain Res, 278315-322.

Weng, Y., Qi, R., Liu, C., Ke, J., Xu, Q., Wang, F., et al. (2017). Disrupted functional connectivity density in irritable bowel syndrome patients. Brain Imaging Behav, 11(6), 1812-1822.

Yu, D., Yuan, K., Luo, L., Zhai, J., Bi, Y., Xue, T., et al. (2017). Abnormal functional integration across core brain networks in migraine without aura. Mol Pain, 13.

Zhang, Y., Ji, G., Li, G., Hu, Y., Liu, L., Jin, Q., et al. (2019). Ghrelin reductions following bariatric surgery were associated with decreased resting state activity in the hippocampus. Int J Obes (Lond), 43(4), 842851.

Zhu, Q., Cai, W., Zheng, J., Li, G., Meng, Q., Liu, Q., et al. (2016). Distinct resting-state brain activity in patients with functional constipation. Neurosci Lett, 632141-146.

\section{Figures}


(A) Key: Correlation

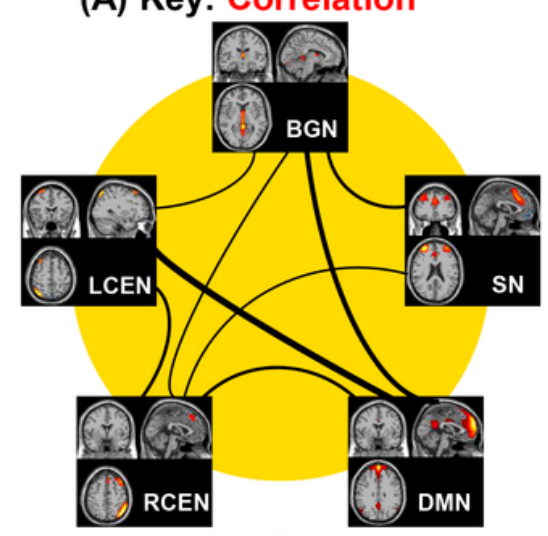

(a) FCon

(B) Key:(a $\longrightarrow$ b: b lags a)

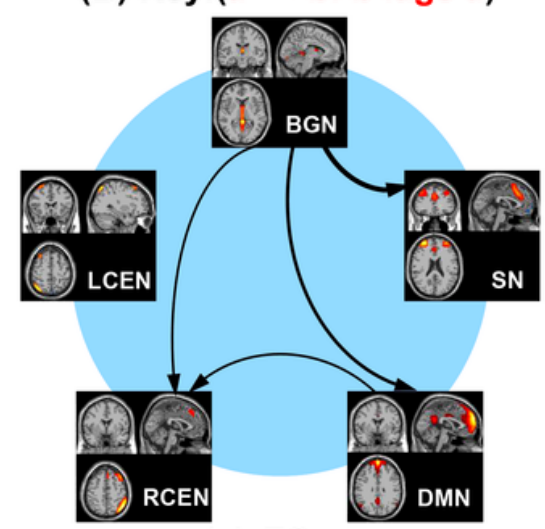

(a) FCon

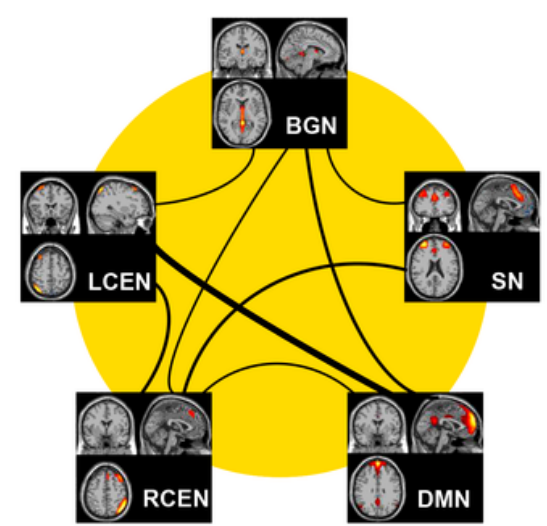

(b) $\mathrm{HC}$

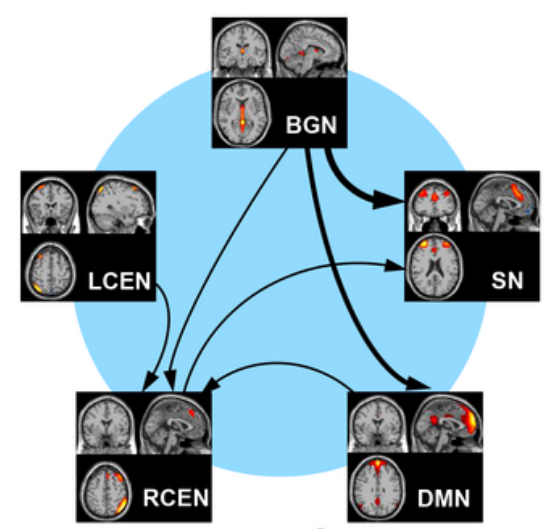

(b) $\mathrm{HC}$

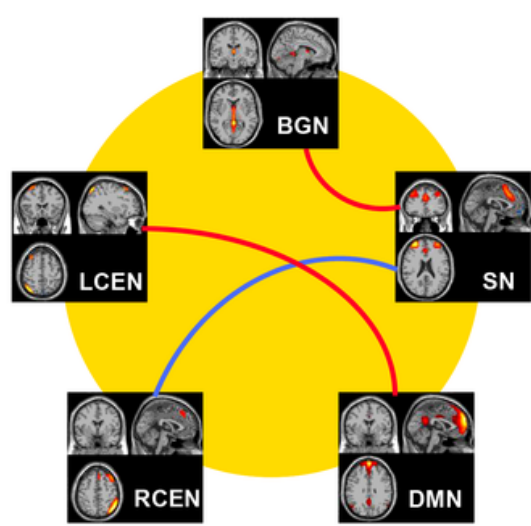

(c) Group difference

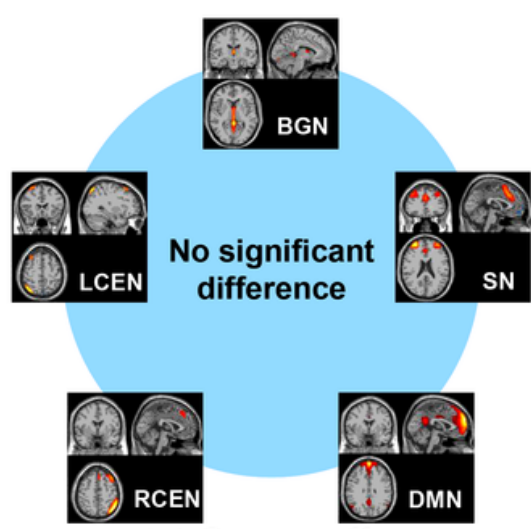

(c) Group difference

\section{Figure 1}

Significant correlations and lags among five RSNs in two groups. (A). Significant correlation between group differences. There were significant differences in correlation in DMN-LCEN (FCon>HC), BGN-SN (FCon>HC), and SN-RCEN (FCon $<\mathrm{HC}$ ) connections between patients with FCon and HC. The red line shows significant connectivity where patients with FCon have a higher correlation than $\mathrm{HC}$, while the blue line shows connectivity where $\mathrm{HC}$ have higher correlation, and the black line represents the correlation of RSNs within each group. Thickness of lines represents the level of correlation strength between RSNs. (B). Significant lag between group differences. The arrow shows the direction of the delay between RSNs. For example, RCEN $\rightarrow$ SN shows RCEN precedes the SN. Thickness of arrow represents the level of correlation strength between RSNs. 


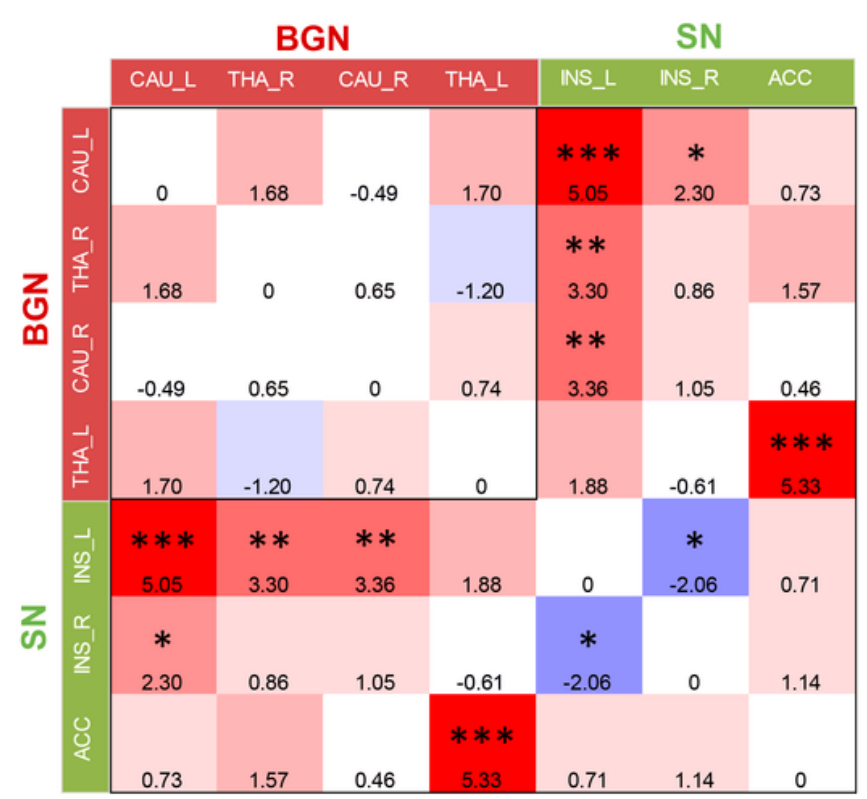

(A)

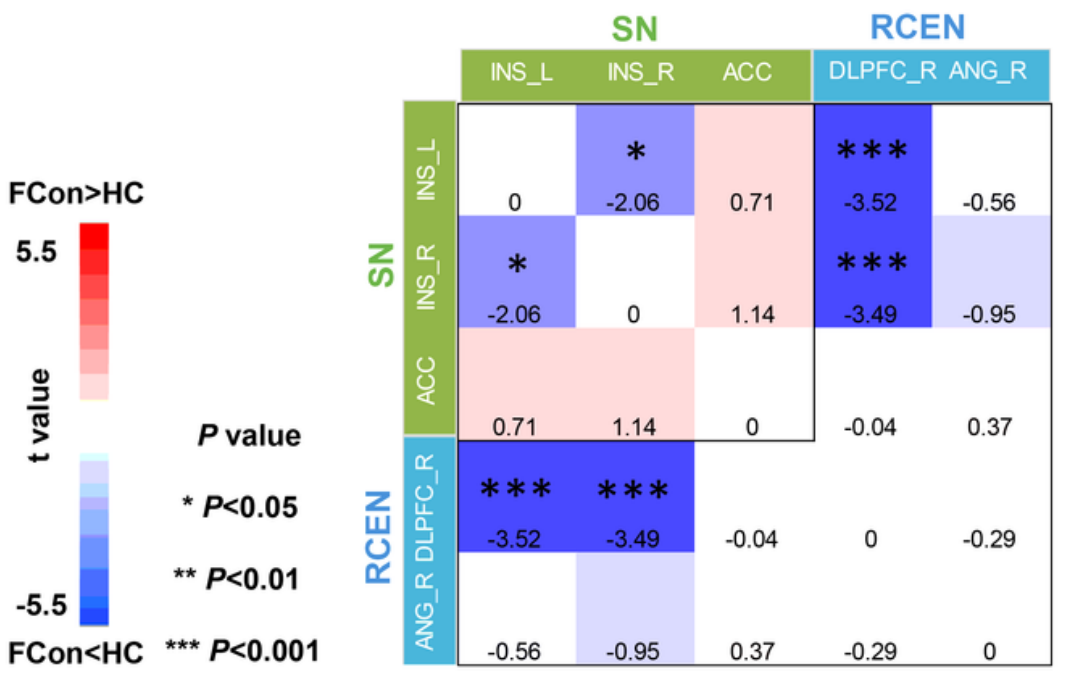

(B)

Figure 2

Two-sample t-test of pairwise FC in BGN-SN and SN-RCEN. The color bar shows the t values. The depth of red and blue color shows the degree of $\mathrm{FCon}>\mathrm{HC} / \mathrm{HC}>\mathrm{FCon}$. The symbol of one asterisk, two asterisks, and three asterisks denotes $P<0.05, P<0.01$, and $P<0.001$. Combined with the $t$ and $P$ value, the darker the blue/red color, the smaller the $P$ value. (A). Two-sample t-test of pairwise FC in BGN-SN. In BGN and SN, the seven ROls were defined. FCon showed higher pair-wise FC of CAU_L-INS_L, THA_R-INS_L, CAU_RINS_L and THA_L-ACC (P<0.002). (B). Two-sample t-test of pairwise FC in SN-RCEN. In SN and RCEN, the five ROls were defined. FCon showed lower paire-wise FC of INS_L-DLPFC_L and INS_R-DLPFC_R $(P<0.005)$. Abbreviation: $F C$, functional connectivity; $B G N$, basal ganglia network; $S N$, salience network; RCEN, right control executive network; FCon, functional constipation; $\mathrm{HC}$, healthy controls; $\mathrm{CAU}$, caudate; THA, thalamus; INS, insula; ANG, angular gyrus; ACC, anterior cingulate cortex; DLPFC, dorsolateral prefrontal cortex. 


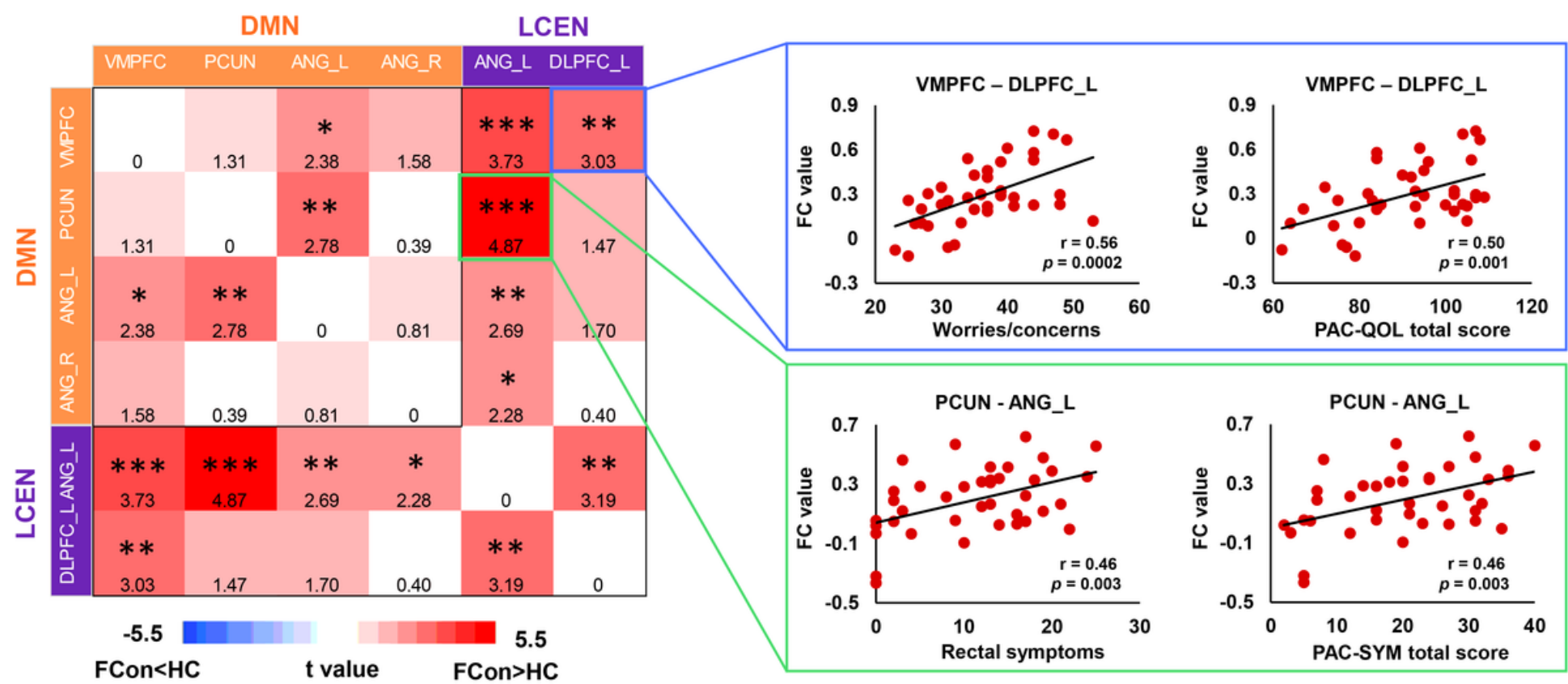

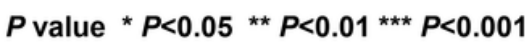

(A)

(B)

Figure 3

. Two-sample t-test of pairwise FC in DMN-LCEN. (A). In the DMN and LCEN, the six ROls were defined. The color bar shows the $t$ values. The depth of red and blue color shows the degree of $\mathrm{FCon}>\mathrm{HC} / \mathrm{HC}>\mathrm{FCon}$. The symbol of one asterisk, two asterisks, and three asterisks denotes $\mathrm{P}<0.05$, $P<0.01$, and $P<0.001$, respectively. Combined with the $t$ and $P$ value, the darker the blue/red color, the smaller the $P$ value. FCon showed higher pair-wise FC of VMPFC-ANG_L, PCUN-ANG_L, and VMPFCDLPFC_L $(P<0.003)$. (B). Correlation analysis between the pairwise FC of DMN-LCEN connections and clinical measures in patients with FCon. FC of VMPFC-left DLPFC was related to the worries/concerns and PAC-QOL total score. Meanwhile, FC strength of PCUN-ANG_L was positively correlated with rectal symptoms and PAC-SYM total score. Abbreviation: FC, functional connectivity; DMN, default mode network; LCEN, left control executive network; VMPFC, ventromedial prefrontal cortex; PCUN, precuneus; ANG, angular gyrus; DLPFC, dorsolateral prefrontal cortex; FCon, functional constipation; PAC-QOL, Patient Assessment of Constipation Quality of Life Scale; PAC-SYM, Patient Assessment of Constipation Symptom Scale; $\mathrm{HC}$, healthy controls. 

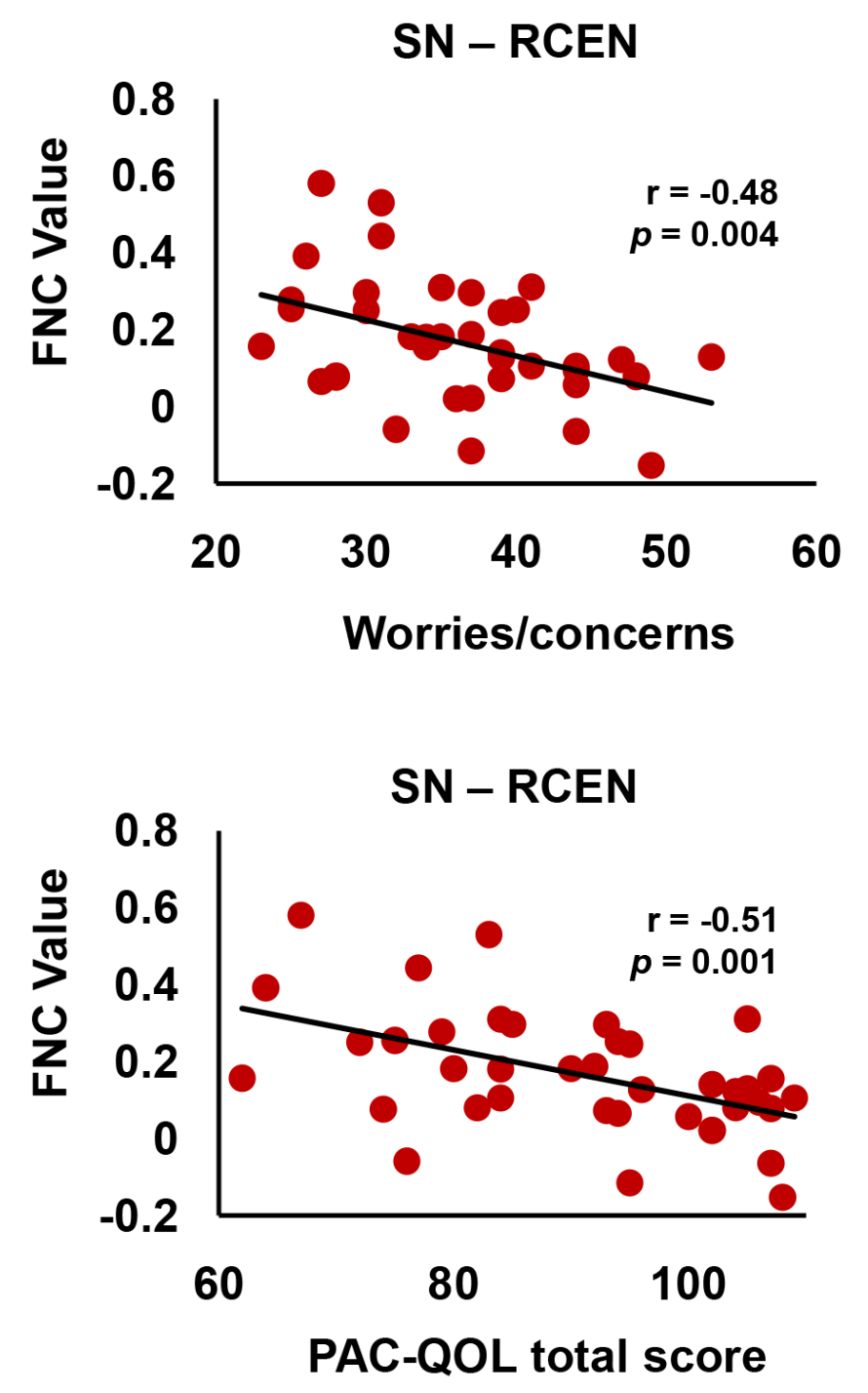
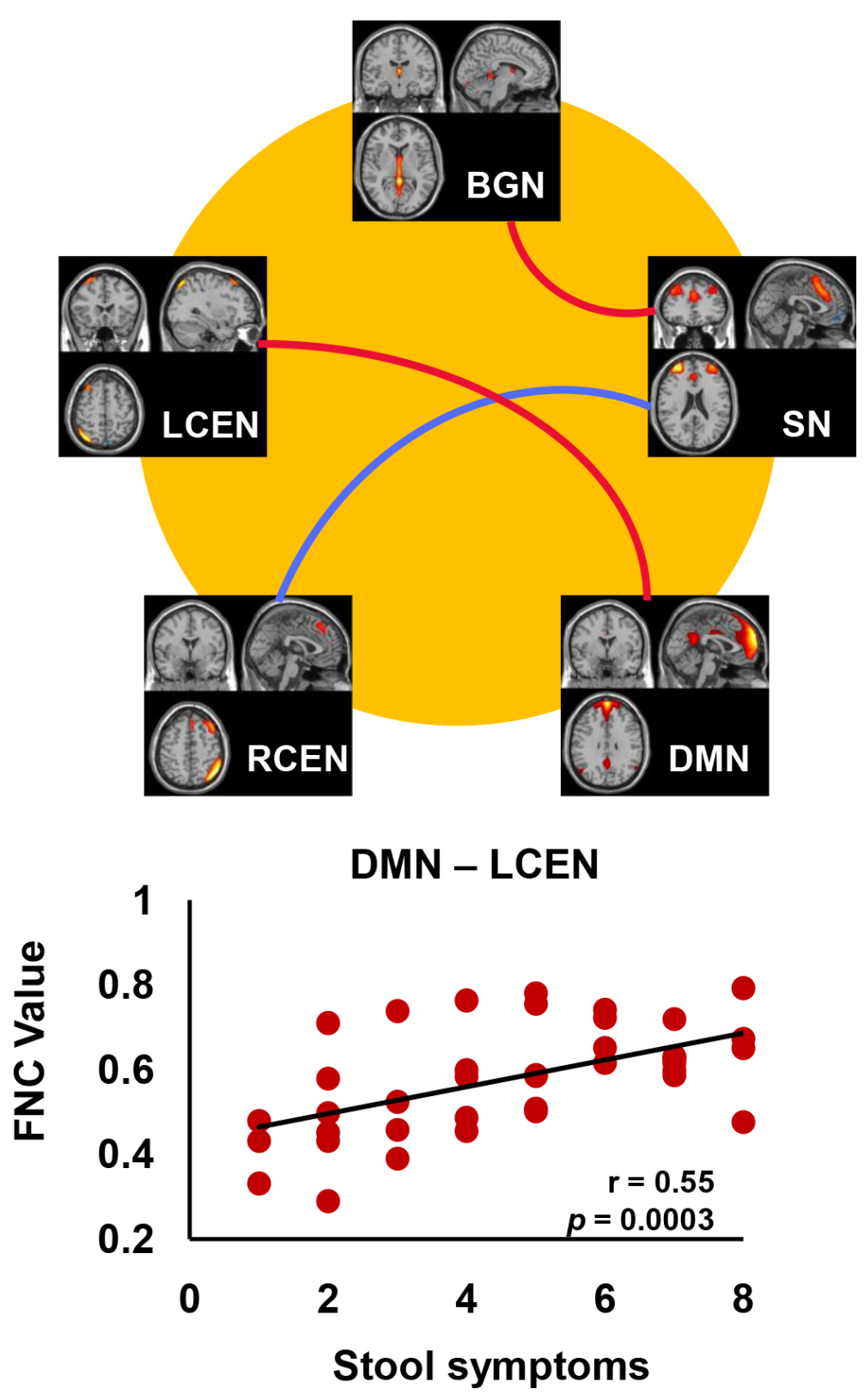

Figure 4

Correlation analysis between FNC strength of RSNs and clinical measures in patients with FCon. FCon had increased FNC of BGN-SN and DMN-LCEN which was positively correlated with stool syndromes, and reduced FNC of SN-RCEN which was negatively correlated with worries/concerns and PAC-QOL total score. Abbreviation: FNC, functional network connectivity; BGN, basal ganglia network; SN, salience network; RCEN, right control executive network; LCEN, left control executive network; DMN, default mode network; FCon, functional constipation; HC, healthy controls; PAC-QOL, Patient Assessment of Constipation Quality of Life Scale. 